\title{
Toward Implementing an MRI-Based PET Attenuation-Correction Method for Neurologic Studies on the MR-PET Brain Prototype
}

Ciprian Catana ${ }^{1}$, Andre van der Kouwe ${ }^{1}$, Thomas Benner ${ }^{1}$, Christian J. Michel², Michael Hamm², Matthias Fenchel ${ }^{2}$, Bruce Fischl ${ }^{1}$, Bruce Rosen ${ }^{1}$, Matthias Schmand ${ }^{2}$, and A. Gregory Sorensen ${ }^{1}$

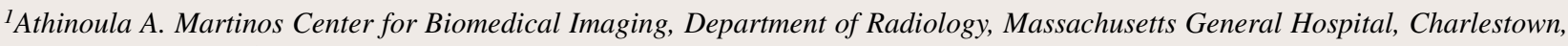
Massachusetts; and ${ }^{2}$ Siemens Healthcare

Several factors have to be considered for implementing an accurate attenuation-correction (AC) method in a combined MR-PET scanner. In this work, some of these challenges were investigated, and an AC method based entirely on the MRI data obtained with a single dedicated sequence was developed and used for neurologic studies performed with the MR-PET human brain scanner prototype. Methods: The focus was on the problem of bone-air segmentation, selection of the linear attenuation coefficient for bone, and positioning of the radiofrequency coil. The impact of these factors on PET data quantification was studied in simulations and experimental measurements performed on the combined MR-PET scanner. A novel dual-echo ultrashort echo time (DUTE) MRI sequence was proposed for head imaging. Simultaneous MR-PET data were acquired, and the PET images reconstructed using the proposed DUTE MRI-based AC method were compared with the PET images that had been reconstructed using a CT-based AC method. Results: Our data suggest that incorrectly accounting for the bone tissue attenuation can lead to large underestimations $(>20 \%)$ of the radiotracer concentration in the cortex. Assigning a linear attenuation coefficient of 0.143 or $0.151 \mathrm{~cm}^{-1}$ to bone tissue appears to give the best trade-off between bias and variability in the resulting images. Not identifying the internal air cavities introduces large overestimations ( $>20 \%$ ) in adjacent structures. On the basis of these results, the segmented CT AC method was established as the silver standard for the segmented MRI-based AC method. For an integrated MR-PET scanner, in particular, ignoring the radiofrequency coil attenuation can cause large underestimations (i.e., $\leq 50 \%$ ) in the reconstructed images. Furthermore, the coil location in the PET field of view has to be accurately known. High-quality bone-air segmentation can be performed using the DUTE data. The PET images obtained using the DUTE MRI- and CT-based $A C$ methods compare favorably in most of the brain structures. Conclusion: A DUTE MRI-based AC method considering all these factors was implemented. Preliminary results suggest

Received Aug. 12, 2009; revision accepted Jun. 14, 2010.

For correspondence or reprints contact: Ciprian Catana, Athinoula A. Martinos Center for Biomedical Imaging, Bldg. 149, Room 2301, 13th St., Charlestown, MA 02129.

E-mail: ccatana@nmr.mgh.harvard.edu

COPYRIGHT $\odot 2010$ by the Society of Nuclear Medicine, Inc. that this method could potentially be as accurate as the segmented CT method and could be used for quantitative neurologic MR-PET studies.

Key Words: simultaneous MR-PET; attenuation correction; ultrashort echo time MRI sequence; brain imaging; head segmentation

J Nucl Med 2010; 51:1431-1438

DOI: 10.2967/jnumed.109.069112

$\mathbf{T}$ here has recently been great interest in combining PET and MRI, and several integrated scanners capable of simultaneous acquisition have been developed and successfully tested for small-animal (1-5) and human (6) imaging. Improved PET data quantification is expected to be a main advantage of MR-PET, and an accurate attenuation-correction (AC) method is necessary for obtaining a precise quantitative measure of the radiotracer concentration. However, because of the limited space available inside the MRI scanner bore, the MRI-compatible PET inserts developed to date are not equipped with a transmission system, the lack of which makes the implementation and validation of an MRI-based AC method necessary. This is, however, not a trivial task because several factors have to be considered to implement an accurate AC method. Because the MRI signal reflects tissue proton densities (and tissue relaxation times) and not electron density, the MR images are not directly related to the tissue linear attenuation coefficients (LACs). However, attenuation maps ( $\mu$-maps) have been previously estimated from segmented MR images (7). Image segmentation is a mature research domain, and many algorithms have been developed to segment images obtained using different imaging modalities, including MRI (8-10). These tasks range from segmentation of a small number of tissue classes such as gray matter (GM) from white matter (WM) to the more complex identification of specific head structures. It is relatively straightforward to identify water- 
based structures using these techniques. The more challenging task consists of differentiating bone tissue from airfilled spaces, because both appear dark (i.e., no signal) on MR images obtained using conventional sequences. Bone is especially relevant as a photon-attenuating medium, being the tissue with the highest LAC and thus likely to introduce large bias in the adjacent GM structures when misclassified as air or soft tissue.

In this work, the challenges and requirements for implementing an MRI-based AC method for the Siemens MRPET brain prototype were investigated. In particular, the need for identifying the bone for accurate PET data quantification in brain structures was studied in simulations using segmented MR and CT images. As a potential solution to the problem of discriminating air from bone on MR images, preliminary results using a new dual-echo ultrashort echo time (DUTE) sequence for imaging bone and discriminating it from air-filled cavities are presented. For the integrated MR-PET scanner, in particular, the challenges related to radiofrequency coil attenuation were also investigated. Proof-of-principle DUTE MRI-based AC was performed on the combined scanner in humans. Our results suggest that it is possible to develop an AC method that could be as accurate as the one provided by the segmented CT AC method using the proposed MRI DUTE sequences.

\section{MATERIALS AND METHODS}

\section{Integrated MR-PET Scanner}

A prototype MRI-compatible PET scanner designed to fit inside the MAGNETOM Trio, a total-imaging-matrix system 3-T human MRI scanner (Siemens Healthcare Inc.), was recently installed at Massachusetts General Hospital. This PET scanner-called BrainPET-uses magnetic field-insensitive avalanche photodiodes as scintillation photon detectors. The inner and outer physical diameters of the gantry are 35 and $60 \mathrm{~cm}$, respectively. The transaxial field of view (FOV) was $32 \mathrm{~cm}$, and the axial FOV was 19.25 $\mathrm{cm}$. Emission data were acquired in list-mode format, sorted in the line-of-response (LOR) space, and compressed axially in the sinogram space for fast reconstruction (11). This axial compression (span, 9; maximum ring difference, 67) generated 1,399 sinograms, each consisting of 256 radial elements and 192 angular projections. The images were reconstructed with the ordinary Poisson ordered-subsets expectation maximization algorithm from prompts, variance-reduced random coincidences (12), detector sensitivity (obtained from a plane source), scatter (13), and attenuation-the latter 2 accounting for the head (different models are investigated in this work) and radiofrequency coil. The reconstructed volume consisted of 153 slices with $256 \times 256$ pixels $(1.25 \times 1.25 \times 1.25 \mathrm{~mm})$. A PET-friendly circularly polarized transmit-8-channel receive radiofrequency coil was specifically designed for this scanner by positioning most of the attenuating components (e.g., capacitors) outside the PET FOV.

\section{Simulations}

Simulations were used to study factors affecting the accuracy of $\mathrm{AC}$ in the combined MR-PET scanner. In particular, the focus was on the bone-air segmentation problem, bone tissue LAC selection, and radiofrequency coil positioning. Tools were developed for generating noiseless emission data with attenuation and for reconstructing the attenuation-corrected images. The emission data were generated in image space, assuming uniform distribution in structures of interest identified from segmented MRI datasets (i.e., GM and WM structures) or assuming uniform distribution in a cylindric phantom. These volumes were then forward projected to obtain the true emission sinograms. True and modeled $\mu$-maps were created in a similar way and were forward projected (and exponentiated) to generate the attenuation-correction factors (ACFs). To obtain the different attenuated emission data, first the true emission sinogram was attenuated using the true AC sinogram. The modeled $\mu$-maps were then used to correct the attenuated emission data. Other factors (e.g., noise, scatter, detector sensitivity, and gaps between detector blocks) were not included in these simulations. The relative changes (RCs) with respect to the true $\mathrm{AC}$ method were calculated for all the voxels included in the initial emission volume using the equation $\mathrm{RC}=100 \times$ $\left(\mathrm{C}_{\text {modeled }}-\mathrm{C}_{\text {true }}\right) / \mathrm{C}_{\text {true }}$, where $\mathrm{C}_{\text {modeled }}$ and $\mathrm{C}_{\text {true }}$ were the values measured from the volumes reconstructed using a particular model or the true AC method, respectively. Small, moderate, large, and severe RCs (i.e., under- and overestimation) were defined as $0 \%-$ $5 \%, 5 \%-10 \%, 10 \%-20 \%$, and greater than $20 \%$, respectively. RC images were used for the qualitative and quantitative analysis of the AC inaccuracy in individual slices. For obtaining a global assessment of the effects, histograms of the RCs for all the voxels included in the initial emission volume were also analyzed in each case. Bias and variability were defined as the deviation from zero (i.e., no RC) of the maximum value observed in the histogram and as the value of the full width at half maximum, respectively.

Bone Tissue Segmentation. Coregistered high-resolution MRI and CT datasets acquired in human subjects were used to segment the brain structures (8) and bone tissue. The PET emission data were created from the segmented MRI data, assuming a 4:1 GMto-WM uptake ratio and no uptake in any of the other structures (e.g., cerebrospinal fluid, scalp, muscles, and fat) (Fig. 1).

Four models of $\mu$-maps were created from the segmented MRI and CT data: 2 MRI-based (MRI first $_{\text {and }}$ MRI second $_{\text {nd }}$ ) anbased $\left(\mathrm{CT}_{\text {segmented }}\right.$ and $\left.\mathrm{CT}_{\text {scaled }}\right)$ (Fig. 1). The first MRI-based

FIGURE 1. Representative slices from simulated datasets obtained from coregistered MRI and CT data. (Left to right) Segmented head structures (brain structures and bone tissues obtained from $\mathrm{MRI}$ and $\mathrm{CT}$, respectively), simulated emission data, and $\mu$-maps $\left(\mathrm{CT}_{\text {scaled, }}\right.$ $\mathrm{CT}_{\text {segmented }}, \mathrm{MRI}_{\text {first }}$, and $\left.M \mathrm{MI}_{\text {second }}\right)$.

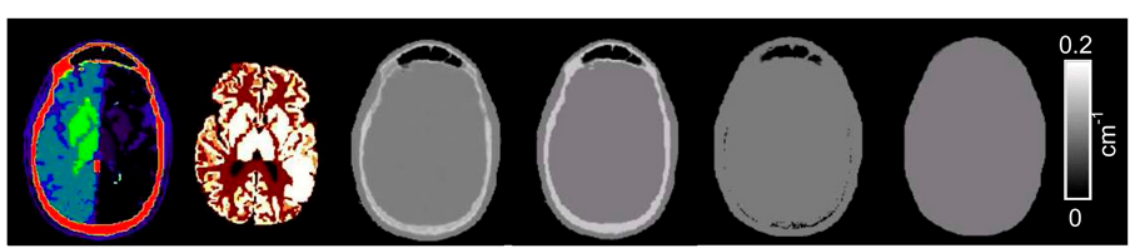




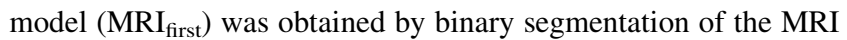
data based on an empirically determined threshold (e.g., $10 \%$ of the maximum intensity), reslicing of the MRI volume so that the MRI voxel size matched the PET voxel size, and assigning of an LAC corresponding to water at $511 \mathrm{keV}$ (i.e., $0.096 \mathrm{~cm}^{-1}$ ) to all nonzero voxels. As a next step (MRI second $_{\text {) }}$, the $\mu$-map obtained in the first step was processed by applying a morphologic closing operation (14) so that all the gaps present in the images (i.e., the voxels corresponding to bone and air cavities) were classified as soft tissue. For the first CT-based method $\left(\mathrm{CT}_{\text {segmented }}\right)$, the $\mathrm{CT}$ dataset was segmented into bone (300-2,000 Hounsfield units [HUs] (15)), soft-tissue ( -200 to $300 \mathrm{HUs})$, and air cavities $(<-200 \mathrm{HUs})$, and uniform LACs were assumed for these 3 tissue classes (i.e., 0.151, 0.096, and $0 \mathrm{~cm}^{-1}$, respectively). For the second CT-based method $\left(\mathrm{CT}_{\text {scaled }}\right)$, the HUs were converted to LACs at $511 \mathrm{keV}$ using the standard PET/CT hybrid scaling method (i.e., all the voxels above and below 300 HUs were scaled using 0.405 and 0.496 as scaling factors, respectively (15)). Four models of ACs (i.e., $\mathrm{MRI}_{\text {first }}, \mathrm{MRI}_{\text {second }}, \mathrm{CT}_{\text {segmented }}$, and $\mathrm{CT}_{\text {scaled }}$ ) were obtained from these maps. The true AC sinogram was derived from the scaled CT data (the current gold standard).

Bone Tissue LAC Selection. Assuming that bone-air segmentation can be performed (using either the DUTE sequences proposed later or an atlas-based method (16)), LACs have to be assigned to all tissue classes identified. However, the value of the bone tissue LAC is still a subject of debate (the published values range from 0.136 to $\left.0.180 \mathrm{~cm}^{-1}\right)(7)$.

Starting from the $\mu$-map derived from the segmented CT dataset described in the "Bone Tissue Segmentation" section, 6 models of $\mu$-maps were created, assuming the following LACs for bone tissue: $0.136,0.143,0.151,0.157,0.171$, and $0.180 \mathrm{~cm}^{-1}$ (7).

Radiofrequency Coil Positioning. Because the probability of both annihilation photons reaching the PET detectors depends on the total attenuation along each particular LOR, another factor that could affect the PET quantification in a combined MR-PET scanner is the attenuation in the radiofrequency coil located between the patient and PET detectors. These effects were studied using a simulated 16-cm-diameter uniform phantom. The selection of the scaling factors was based on the analysis of the data obtained from CT and from 2 transmission scans of the radiofrequency coils (at 662 and $511 \mathrm{keV}$ on the Siemens HRRT and $\mathrm{HR}+$, respectively). The complete $\mu$-map was obtained by assuming a constant $0.096 \mathrm{~cm}^{-1} \mathrm{LAC}$ throughout the phantom volume and adding it to the coil $\mu$-map. Because the coil is actually larger than the reconstructed PET FOV, the forward projection was performed on an extended FOV larger than the coil diameter. The central 256 radial bins of the attenuation sinograms were subsequently cropped for each angle to obtain the AC sinograms required by the data processing. Images were reconstructed from the data including and ignoring the radiofrequency coil $\mu$-map. Furthermore, to study the effect of coil mispositioning on the PET quantification, the coil $\mu$-map was translated $1-5 \mathrm{~mm}$ along the $x$ - and $y$-axes and rotated $1^{\circ}-5^{\circ}$ about the $z$-axis.

\section{Phantom and Healthy Volunteer Studies}

Experimental measurements were performed to study some of these factors using the standard data acquisition, processing, and reconstruction methods on the combined MR-PET scanner. The purpose of these experimental measurements was not to replicate the simulations but rather to provide potential solutions in each case for this particular scanner. All the human studies were approved by the Institutional Review Board at Massachusetts General Hospital.

Bone Tissue Imaging Using Ultrashort Echo Time (UTE) Sequences. UTE sequences have been recently proposed and developed for imaging cortical bone and other connective tissues of relevance for musculoskeletal system imaging $(17,18)$. These sequences hold the potential of being an elegant solution to the MRI bone-air segmentation problem and for deriving the $\mu$-maps (19). These sequences are used to image tissues with short T2* relaxation time, such as bone (T2* in the range of $0.05-2 \mathrm{~ms}$ ). In principle, the bone tissue could be identified if data were acquired at 2 echo times such that the signal from the bone is present in the first-echo $\left(\mathrm{UTE}_{1}\right)$ and not in the second-echo $\left(\mathrm{UTE}_{2}\right)$ dataset, whereas the signals from other tissue classes (i.e., soft tissue and air) are similar in both cases. These sequences were tested trying to identify the parameters that are most useful for bone-air discrimination.

To minimize the acquisition time and overcome the potential image artifacts introduced by patient motion when using sequential UTE, new DUTE sequences were proposed and implemented for collecting the signal from both echoes during the same acquisition. The following parameters were chosen for these studies: echo time, $0.07 / 2.46 \mathrm{~ms}$; repetition time, $200 \mathrm{~ms}$; flip angle, $10^{\circ}$; radial projections, 32,000; bandwidth, 1,532 Hz/pixel; FOV, $320 \mathrm{~mm}$; base resolution, 192; and acquisition time, $3 \mathrm{~min}$ $20 \mathrm{sec}$. As proof of principle, a human skull was first imaged using this DUTE sequence and compared with the results from the CT (120 kV/50 mA, 20-s acquisition time). Next, human volunteers were scanned inside the BrainPET scanner.

Bone Tissue LAC Estimation. To estimate the bone tissue LAC, 325 CT datasets acquired at Massachusetts General Hospital were analyzed. All the subjects were scanned on the same scanner (GE Healthcare) using the same acquisition protocol (i.e., 2.5-mm bone axials, $120 \mathrm{kVp} / 250 \mathrm{~mA}$ ). For each dataset, the average values and SD for all the voxels in the 300 - to $2,000-\mathrm{HU}$ range were computed and these data were analyzed as a function of sex and age.

Radiofrequency Coil Positioning. The position of the radiofrequency coil with respect to the BrainPET scanner was determined by placing fiducial markers filled with a solution of ${ }^{18}$ F-FDG at precise locations on the coil and acquiring PET data. To test the reproducibility of the coil positioning with respect to the PET scanner, the whole setup was repeatedly repositioned inside the scanner. In addition, the PET insert was removed and repositioned inside the magnet (as done routinely at our institution), maintaining the coil in the same location. PET data were acquired in each case, and the positions of the fiducial markers in the reconstructed images were compared.

\section{Simultaneous MR-PET Human Studies}

Four subjects were scanned for these proof-of-principle studies. In all these cases, the head $\mu$-map was derived by analyzing the data obtained with the DUTE sequence. Rather than using the simple difference between the first echo $\left(\mathrm{DUTE}_{1}\right)$ and the second echo (DUTE ${ }_{2}$, we applied several steps for improving the identification of the 3 classes (i.e., soft tissue, bone, and air) and generating the $\mu$-maps

For soft tissue, a mask that included the voxels from all classes was obtained by applying a morphologic closing operation to the DUTE 2 data. This step was required for excluding the voxels outside the subject's head (e.g., radiofrequency coil). Subsequently, an opening operation was applied to exclude 
voxels at the air-tissue interface, where susceptibility artifacts were present.

For bone tissue, the original DUTE ${ }_{1}$ and DUTE $_{2}$ volumes were first divided by the corresponding smoothed volumes obtained after applying a 3-dimensional gaussian low-pass filter with a 20-mm-radius kernel. In this way, the image inhomogeneities due to coil nonuniform sensitivity were removed. The resulting datasets were combined using the transformation (DUTE ${ }_{1}-$ $\left.\mathrm{DUTE}_{2}\right) /\left(\mathrm{DUTE}_{2}{ }^{2}\right)$ tuned to enhance the bone tissue voxels. Specifically, the numerator allows the selection of the voxels with the largest signal change (i.e., bone vs. other classes), whereas the denominator allows the selection of the voxels with the lowest signal in the $\mathrm{DUTE}_{2}$ images (i.e., bone and air). Bone LAC $\left(0.151 \mathrm{~cm}^{-1}\right)$ was assigned to all the voxels above an empirically determined threshold from the transformed images (i.e., 0.012).

For air cavities, a procedure similar to the one used for bone tissue was applied to segment the voxels corresponding to air cavities. We started from the low-pass filtered data combined using the transformation $\left(\mathrm{DUTE}_{1}+\mathrm{DUTE}_{2}\right) /\left(\mathrm{DUTE}_{1}^{2}\right)$ and an empirically determined threshold from the transformed images (i.e., 0.14).

Soft-tissue LAC was assigned to all the voxels included in the mask that were not identified as bone or air.

An identical data-processing workflow and the same thresholds were used for deriving the DUTE-segmented $\mu$-maps in all 4 subjects. CT data were also available and were used to generate the CT-derived $\mu$-maps ( $\mathrm{CT}_{\text {scaled }}$ and $\mathrm{CT}_{\text {segmented }}$ methods). The CT and MR-PET studies were performed less than 1 mo apart, and no surgical procedures that would alter the morphology of the skull or brain were performed between the scans. Because the MRI and PET volumes were already coregistered, the CT volume was coregistered to the MRI volume.

For all subjects, the complete $\mu$-maps were obtained by adding the DUTE- or CT-segmented head $\mu$-maps to the radiofrequency coil $\mu$-map. The PET data reconstructed using the ACFs obtained from these $\mu$-maps were qualitatively and quantitatively compared.

\section{RESULTS}

\section{Simulations}

Bone Tissue Segmentation. The air-bone tissue segmentation problem is approached differently in the 2 approximate MRI-based AC methods. In the $\mathrm{MRI}_{\text {first }}$ model, the bone tissue is misclassified as air, whereas in the $\mathrm{MRI}_{\text {second }}$ model the voxels corresponding to bone tissue and some thin air cavities are misclassified as water. Both methods introduce biases in the reconstructed images, and representative RC images (and histograms) are shown in Figure 2A and Supplemental Figure 1A (supplemental materials are available online only at http://jnm.snmjournals.org). The $\mathrm{MRI}_{\text {first }}$ method leads to moderate overall underestimation of the activity and large underestimation in structures adjacent to bone. The MRI $\mathrm{I}_{\text {second }}$ method leads to a moderate overall underestimation in most brain structures but also to a severe overestimation in structures adjacent to real air cavities filled by the morphologic filter. The $\mathrm{CT}_{\text {segmented }}$ model estimates accurately (within 5\%, compared with the $\mathrm{CT}_{\text {scaled }}$ model) the activity concentrations in most of the brain structures.

Bone Tissue LAC Selection. Representative RC images (and histograms) for 3 of the $6 \mathrm{AC}$ models studied are
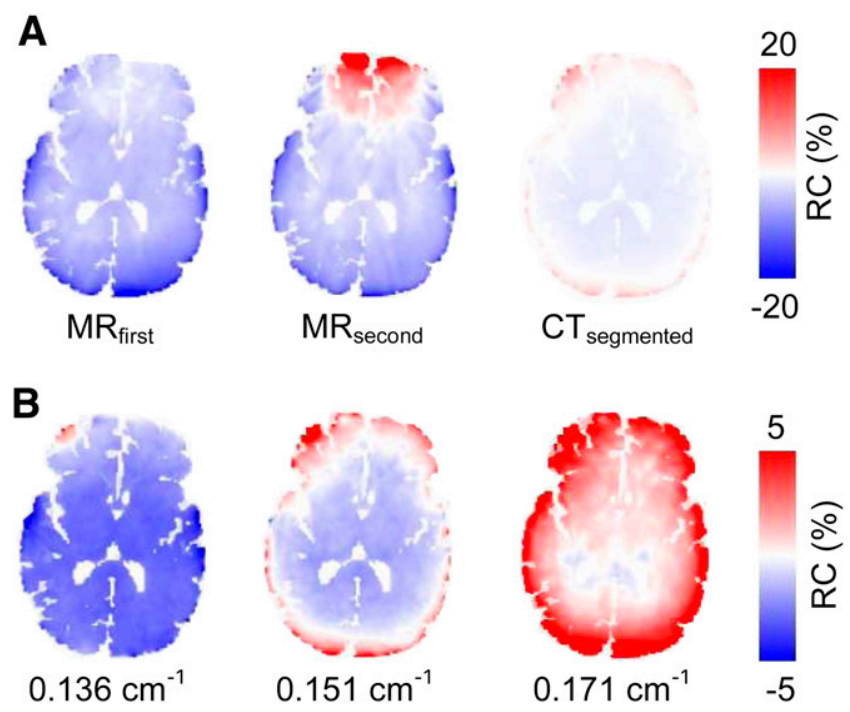

$-5$

FIGURE 2. Subject-related factors affecting PET data quantification: effects of bone tissue segmentation $(A)$ and bone LAC selection (B). Representative images of RCs are presented for each case.

shown in Figure 2B and Supplemental Figure 1B. Overall moderate under- and overestimations were observed for the lower and higher LAC values, especially in structures adjacent to bone. The LACs that resulted in the smallest image bias and smallest variability were 0.171 and $0.136 \mathrm{~cm}^{-1}$, respectively. However, the former had the largest variability and the latter had the largest bias. Assigning LACs of 0.143 and $0.151 \mathrm{~cm}^{-1}$ resulted in a better trade-off between bias and variability.

Radiofrequency Coil Positioning. A CT scan of the radiofrequency coil and $\mathrm{RC}$ images (and histograms) for representative cases studied are shown in Figure 3 and Supplemental Figure 1C. Large underestimations were observed in the data of the simulated phantom, reconstructed ignoring the coil $\mu$-map. The results of the coil $\mu$-map displacement studies suggest that for this particular coil, translations of $1-2 \mathrm{~mm}$ in the $x$ or $y$ directions or rotations of $1^{\circ}-2^{\circ}$ about the $z$-axis can be tolerated without introducing significant changes in the reconstructed PET images (i.e., $<5 \%$ change for all the cases). Similar to the previous case, larger variability and no changes in bias were observed for larger displacements. Displacements larger than $5 \mathrm{~mm}$ or $5^{\circ}$ are not possible in the case of the 8-channel coil that was custom-built to fit tightly inside the BrainPET scanner.

\section{Phantom and Healthy Volunteer Studies}

Bone Tissue Imaging Using UTE Sequences. Representative slices obtained using the single-echo UTE sequences are shown in Figure 4A. Bone signal intensity shows significant changes when the data are acquired with an echo time of $0.07 \mathrm{~ms}$, compared with the data acquired with an echo time of $2.46 \mathrm{~ms}$, whereas the air regions show no significant change. The acquisition times for these sequences 


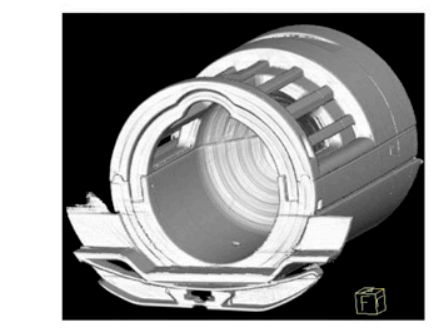

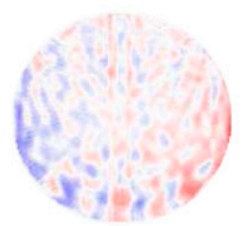

$3 \mathrm{~mm}$ trans. along $x$-axis

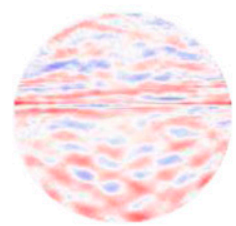

$3 \mathrm{~mm}$ trans. along $y$-axis

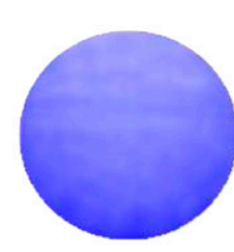

No coil AC

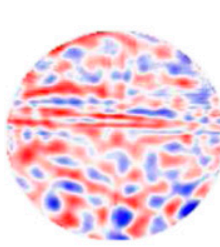

$3^{\circ}$ rot. about $z$-axis

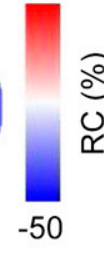

5

อ ญ
FIGURE 3. Coil-related factors affecting PET data quantification of simulated cylinder: CT of radiofrequency coil (top left) and representative images of RCs observed when coil attenuation is ignored (top right) or inaccurately accounted for (bottom row). rot. = rotation; trans. = translation.

were $4.5 \mathrm{~min}$ for a single average. Because the signals from the short and long $\mathrm{T} 2 *$ components were acquired separately, the total acquisition time was 9 min. Our goal was to spend minimal time acquiring the data used for deriving the $\mu$-map so that other MRI sequences could be run simultaneously with the PET data acquisition. A solution to this problem is the DUTE sequence proposed, which has the advantage of eliminating potential motion artifacts that could occur when using sequential UTE sequences. This is particularly relevant because the segmentation accuracy depends on the relationship between the 2 signals for each voxel. Figure 4B shows 3-dimensional reconstructions of the skull obtained from the DUTE $_{1}$ (left) and CT data (right). These images were registered closely within the resolution of the DUTE data, suggesting negligible distortions, compared with CT for PET AC purposes.

Bone Tissue LAC Estimation. Small variations with age and sex were noted in the average values determined from the analysis of the $325 \mathrm{CT}$ datasets, but the differences observed were not statistically significant (Table 1). The overall average value was 1,220 HUs (SD, $24 \mathrm{HUs}$ ). A $0.143 \mathrm{~cm}^{-1}$ LAC was obtained by converting this average value to $\mathrm{LAC}$ at $511 \mathrm{keV}$ and using the same scaling factor for bone tissue as that used in the simulations. Interestingly, this was also 1 of the 2 values that provided the best tradeoff between bias and variability in our simulations.

Radiofrequency Coil Positioning. The displacements measured after the radiofrequency coil and PET insert were repeatedly repositioned inside the scanner were smaller than $1 \mathrm{~mm}$ along and $1^{\circ}$ about all the axes. The results of our simulations suggest that these displacements are not expected to cause major changes in the reconstructed images. However, problems may arise when dedicated
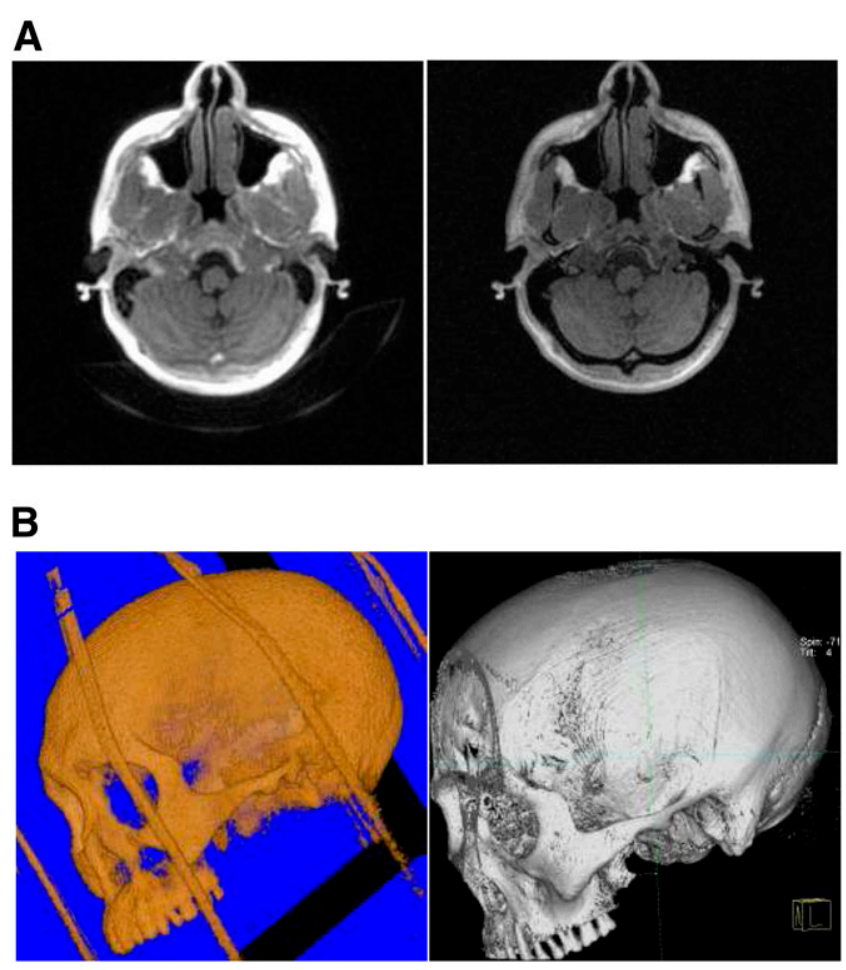

FIGURE 4. Bone MRI using single-echo UTE sequences. (A) Representative images obtained in healthy volunteer: $\mathrm{UTE}_{1}$ (left) and $\mathrm{UTE}_{2}$ (right). (B) Human skull imaging: MRI DUTE $_{1}$ image ( $\sim 1 \mathrm{~mm}$ isotropic) (left) and CT scan $(200 \mu \mathrm{m}$ isotropic) (right). MR image also shows location of radiofrequency coil elements relative to skull.

(flexible) surface coils are used, and the DUTE sequences might be useful for determining their location. As Figure 4B (left) demonstrates, in cases for which the coil position or shape (in the case of flexible coils) is unknown, the DUTE $_{1}$ data might be used for localization.

\section{Simultaneous MR-PET Human Studies}

The $\mu$-maps generated from the segmented $\mathrm{CT}$ and DUTE data (Supplemental Fig. 2A) are shown in Figure 5A for a representative subject (CT and DUTE data, left and right, respectively) and in Supplemental Figures 3A-3C for the other 3 subjects. These images demonstrated a good overall agreement, with most of the soft tissue, bone, maxillary sinus, and mastoid air cavities being correctly segmented. The skull appeared slightly thinner in the DUTE-based segmentation, as is explained by the relatively poor spatial resolution of the DUTE data (i.e., $1.67 \mathrm{~mm}$ ). The segmentation method failed to identify some parts of the nasal air cavities; these voxels were misclassified as bone. Similarly, a small fraction of the cerebrospinal fluid voxels (which had low signal in the original data) was misclassified as bone or air. Adding the information derived from another sequence (e.g., magnetization-prepared rapid-acquisition gradient-echo) could be a solution to these problems but would increase the complexity of the method. Instead, the focus will be on improving the DUTE sequence itself (e.g., higher spatial 


\begin{tabular}{|c|c|c|c|c|c|c|}
\hline \multirow[b]{2}{*}{ Age (decade) } & \multicolumn{3}{|c|}{ Men } & \multicolumn{3}{|c|}{ Women } \\
\hline & No. of subjects & $\mathrm{SD}(\mathrm{HU})$ & Mean $(\mathrm{HU})$ & No. of subjects & $\mathrm{SD}(\mathrm{HU})$ & Mean $(\mathrm{HU})$ \\
\hline 0 & 4 & 18 & 1,220 & 0 & NA & NA \\
\hline 1 & 5 & 19 & 1,198 & 4 & 31 & 1,205 \\
\hline 2 & 18 & 30 & 1,178 & 16 & 24 & 1,207 \\
\hline 3 & 16 & 18 & 1,230 & 12 & 17 & 1,223 \\
\hline 4 & 36 & 25 & 1,232 & 19 & 31 & 1,211 \\
\hline 5 & 28 & 27 & 1,227 & 19 & 22 & 1,245 \\
\hline 6 & 22 & 26 & 1,251 & 24 & 20 & 1,247 \\
\hline 7 & 15 & 32 & 1,215 & 28 & 25 & 1,235 \\
\hline 8 & 12 & 23 & 1,232 & 26 & 31 & 1,213 \\
\hline 9 & 8 & 21 & 1,210 & 5 & 22 & 1,179 \\
\hline 10 & 4 & 10 & 1,221 & 4 & 17 & 1,230 \\
\hline
\end{tabular}

resolution, increased bandwidth, larger number of radial projections) and refining the segmentation, the goal being to develop a method that relies solely on the DUTE data.

Images from the PET data corrected using the ACFs derived from the CT- and DUTE-segmented $\mu$-maps are shown in Figure 5B. These data suggest that the visual interpretation of the PET images would not be impaired

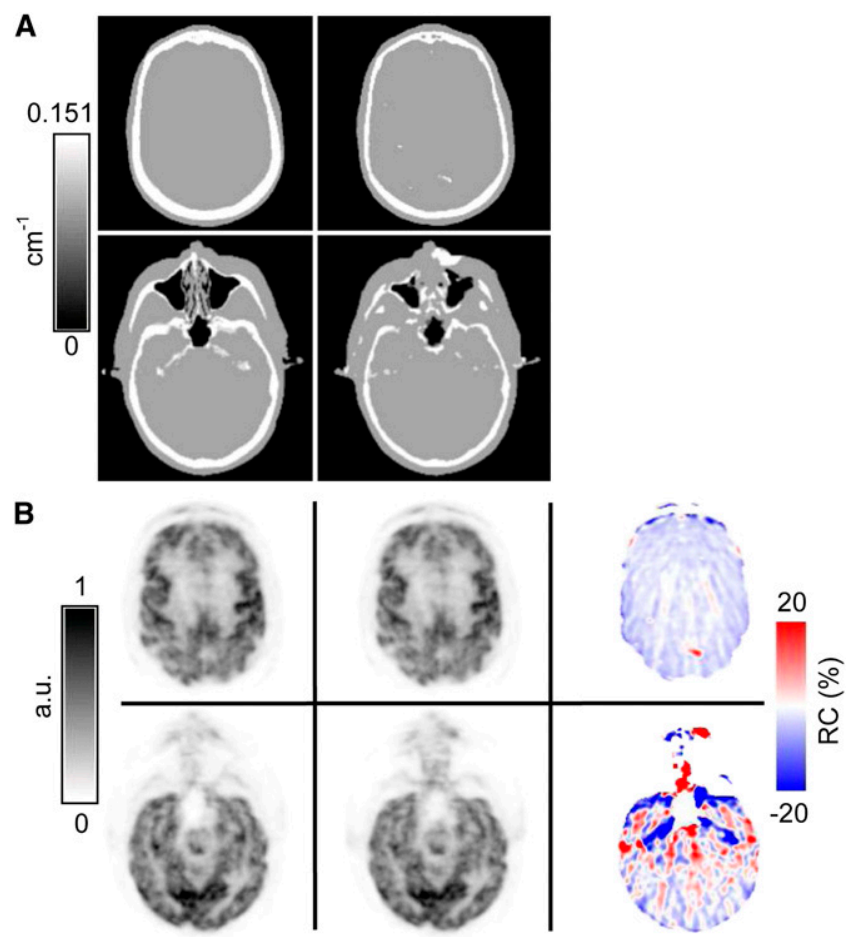

FIGURE 5. Proof-of-principle DUTE-based AC for integrated MR-PET scanner. (A) $\mu$-maps obtained from segmentation of 3 tissue classes based on CT (left) and DUTE (right) data. (B) PET images reconstructed using CT-based (left) and DUTE-based (middle) AC methods and RC images between the 2 methods (right). Images at 2 representative locations are shown in each case. a.u. = arbitrary units. using the DUTE-based AC method as currently implemented. However, as observed from the RC images (using the segmented CT scan as a reference), the data still contained moderate to severe under- and overestimations and they corresponded to the misclassified voxels in the $\mu$-map (Fig. 5B).

Representative PET images reconstructed ignoring and including the coil attenuation are shown in Figure 6. In addition to the severe underestimation of the activity (Fig. 6 , middle column), axial artifacts can be observed in the images (Fig. 6, sagittal). These artifacts disappeared after accounting for the coil attenuation (Fig. 6, right column).

\section{DISCUSSION}

Several factors have to be considered when one is implementing an MRI-based PET AC method for quantitative imaging with the BrainPET scanner.

Assuming that the radiofrequency coil attenuation is properly accounted for, our results show that the next factor that could have the largest impact on PET data quantification is bone-air segmentation. As demonstrated by our simulations, the $\mathrm{MRI}_{\text {frst }}$ method that was used in our initial MRPET studies was clearly inaccurate because it ignored bone attenuation. The MRI second $_{\text {method partially accounted for }}$ this attenuation, misclassifying bone as water; however, some voxels corresponding to air-filled cavities were also treated similarly and, as a result, bias was introduced in the reconstructed images. Nevertheless, the MRI ${ }_{\text {second }}$ method is likely better than most calculated AC methods (e.g., ellipse, contour fitting in image or sinogram space $(20-23)$. These methods have not been designed to segment the internal air cavities (e.g., nasal, oral, mastoid, and frontal sinus). This nonsegmentation is relevant for most currentgeneration PET scanners using calculated AC, including the BrainPET, because the subject's head is not in the traditional anterior commissure-posterior commissure orientation and a large fraction of the LORs are passing through air cavities. Although not specifically studied in our simulations, more severe overestimations of activity in the brain 


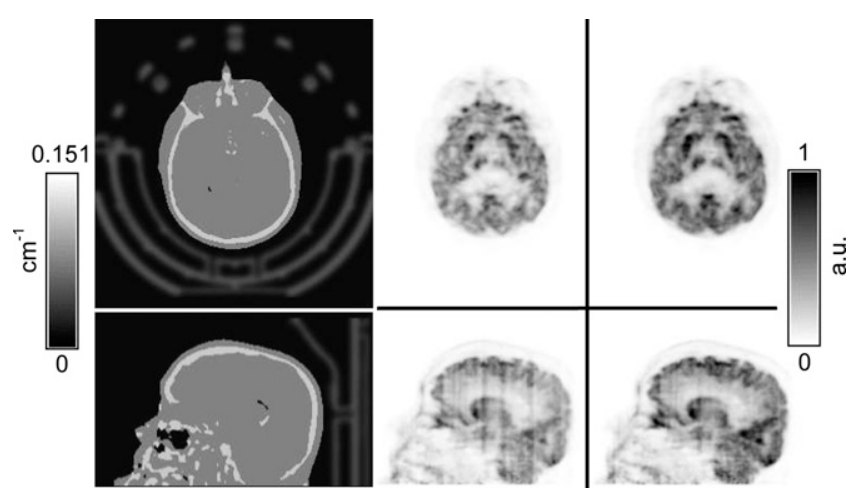

FIGURE 6. PET images reconstructed ignoring (middle column) and including (right column) radiofrequency coil attenuation. Representative slices in 2 orientations are shown in each case. Corresponding slices of complete attenuation map including coil are shown in left column. a.u. = arbitrary units.

regions adjacent to air cavities would likely be observed in the case of the calculated methods. The under- and overestimation effects could even cancel each other in regions adjacent to mixed air-bone structures (e.g., frontal sinus or mastoid bone), making interpretation of the images even more difficult.

The scaled CT method (15) is considered the gold standard for deriving the $\mu$-map in clinical PET/CT scanners. Currently, the $\mathrm{CT}_{\text {segmented }} \mathrm{AC}$ method is used only for selective segmentation of voxels corresponding to contrast agents and metallic implants. More important, this method is likely the silver standard for segmented MRI-based AC methods. The results of the simulations presented demonstrate that an accurate delineation of bone is necessary for brain studies using combined MR-PET scanners and that a method to precisely identify bone is essential, because the GM is so close to the skull. Although atlas-based methods could also be used $(16,24,25)$, in patients with modified bone anatomy (i.e., glioblastoma patients) the DUTE approach (possibly incorporating some atlas-based information) may be superior.

Misregistration between the emission volume and $\mu$-map is a common source of artifacts for all AC methods and is usually caused by subject motion. Although MRI and PET are acquired simultaneously, multiple MRI sequences are generally used, and motion may occur during the acquisition. These effects can be reduced on an integrated MR-PET scanner. First, the hardware coregistration is likely to be superior to the software coregistration of the separately acquired datasets. More important, the temporal correlation of the PET and MRI signals allows one to use the MRI signal for tracking the motion of the object and for correcting the PET data retrospectively (26). There are other factors that could affect the spatial coregistration. Any deformations present in the MRI data (due to MRI distortions caused by gradient nonlinearities) have to be characterized and corrected for. Dental implants could also introduce artifacts in the MRI-based segmentation, as they do in CT. However, instead of showing as high-intensity streaks as in CT, the artifacts are more localized and appear as round signal voids in MRI. Of relevance to the DUTE method proposed, these artifacts are reduced in the DUTE 1 images.

Knowing the exact position (i.e., within a few millimeters) of the radiofrequency coil with respect to the magnet isocenter is not essential for MRI; however, positioning becomes relevant in a combined MR-PET scanner. Our data demonstrate that coil attenuation cannot be ignored. Our coil has been redesigned to fulfill minimum attenuation requirements.

Finally, PET data accuracy may be affected by reconstruction-related artifacts. Of particular interest in the case of the BrainPET prototype are the transaxial gaps between the block detectors because they can concentrate any data inconsistencies due to imperfect normalization, attenuation, and scatter correction. This affect explains the axial artifacts observed when the radiofrequency coil attenuation and scatter were neglected, as illustrated in Figure 6. The characterization of these inconsistencies is, however, outside the scope of the present paper.

\section{CONCLUSION}

Attenuation correction is a mandatory step not only for obtaining quantitative data but also for performing meaningful qualitative image interpretation in PET studies. In this work, segmented CT was proposed as the silver standard for segmented MRI-based AC. For accurate AC in neurologic studies performed using the integrated MRPET scanner, 3 compartments must be identified: waterbased structures (i.e., WM, GM, and cerebrospinal fluid), bone tissue (i.e., skull), and air-filled cavities. The most challenging task-the discrimination of bone tissue from air cavities - can be achieved using the proposed DUTE sequence. In addition, the attenuation of the radiofrequency coil has to be accounted for. An MRI DUTE-based AC method that considers all these aspects could, in principle, provide an estimation of the radiotracer concentration in a particular voxel as accurately as could the silver standard. Implementing an accurate MRI-based AC method is essential for the wide acceptance of this new imaging modality and will allow us to take advantage of the augmented quantitative capabilities of the combined MR-PET scanner.

\section{ACKNOWLEDGMENTS}

We gratefully acknowledge Larry Byars from Siemens for many extremely useful discussions and for his invaluable contribution to the MR-PET project. This work was partly supported by NIH grant 1R01CA137254-01A1.

\section{REFERENCES}

1. Catana C, Procissi D, Wu Y, et al. Simultaneous in vivo positron emission tomography and magnetic resonance imaging. Proc Natl Acad Sci USA. 2008; 105:3705-3710. 
2. Judenhofer MS, Wehrl HF, Newport DF, et al. Simultaneous PET-MRI: a new approach for functional and morphological imaging. Nat Med. 2008;14: 459-465.

3. Raylman RR, Majewski S, Velan SS, et al. Simultaneous acquisition of magnetic resonance spectroscopy (MRS) data and positron emission tomography (PET) images with a prototype MR-compatible, small animal PET imager. J Magn Reson. 2007;186:305-310.

4. Lucas AJ, Hawkes RC, Ansorge RE, et al. Development of a combined microPET-MR system. Technol Cancer Res Treat. 2006;5:337-341.

5. Woody C, Schlyer D, Vaska P, et al. Preliminary studies of a simultaneous PET/ MRI scanner based on the RatCAP small animal tomograph. Nucl Instrum Methods Phys Res Sect A. 2007;571:102-105.

6. Schlemmer H-PW, Pichler BJ, Schmand M, et al. Simultaneous MR/PET imaging of the human brain: feasibility study. Radiology. 2008;248:1028-1035.

7. Zaidi H, Montandon ML, Slosman DO. Magnetic resonance imaging-guided attenuation and scatter corrections in three-dimensional brain positron emission tomography. Med Phys. 2003;30:937-948.

8. Fischl B, Salat DH, Busa E, et al. Whole brain segmentation: automated labeling of neuroanatomical structures in the human brain. Neuron. 2002;33:341-355.

9. Zaidi H, Ruest T, Schoenahl F, Montandon ML. Comparative assessment of statistical brain MR image segmentation algorithms and their impact on partial volume correction in PET. Neuroimage. 2006;32:1591-1607.

10. Fischl B, Salat DH, van der Kouwe AJW, et al. Sequence-independent segmentation of magnetic resonance images. Neuroimage. 2004;23(suppl 1):S69-S84.

11. Hong IK, Chung ST, Kim HK, Kim YB, Son YD, Cho ZH. Ultra fast symmetry and SIMD-based projection-backprojection (SSP) algorithm for 3-D PET image reconstruction. IEEE Trans Med Imaging. 2007;26:789-803.

12. Byars LG, Sibomana M, Burbar Z, et al. Variance reduction on randoms from coincidence histograms for the HRRT. IEEE Nucl Sci Symp Conf Rec. 2005;5: 2622-2626.

13. Watson CC. New, faster, image-based scatter correction for 3D PET. IEEE Trans Nucl Sci. 2000;47:1587-1594.
14. Serra J. Image Analysis and Mathematical Morphology. London, U.K.: Academic Press; 1982.

15. Kinahan PE, Hasegawa BH, Beyer T. X-ray-based attenuation correction for positron emission tomography/computed tomography scanners. Semin Nucl Med. 2003;33:166-179.

16. Hofmann M, Steinke F, Scheel V, et al. MRI-based attenuation correction for PET/MRI: a novel approach combining pattern recognition and atlas registration. J Nucl Med. 2008;49:1875-1883.

17. Reichert ILH, Robson MD, Gatehouse PD, et al. Magnetic resonance imaging of cortical bone with ultrashort TE pulse sequences. Magn Reson Imaging. 2005;23: 611-618.

18. Robson MD, Bydder GM. Clinical ultrashort echo time imaging of bone and other connective tissues. NMR Biomed. 2006;19:765-780.

19. Keerman V, Vandenberghe S, De Deene Y, Luypaert R, Broux T. MR-based attenuation correction for PET using an ultrashort echo time (UTE) sequence. IEEE Nucl Sci Symp Conf Rec. 2009:3929-3934.

20. Michel C, Bol A, Devolder AG, Goffinet AM. Online brain attenuation correction in PET: towards a fully automated data handling in a clinical environment. Eur $J$ Nucl Med. 1989;15:712-718.

21. Siegel S, Dahlbom M. Implementation and evaluation of a calculated attenuation correction for PET. IEEE Trans Nucl Sci. 1992;39:1117-1121.

22. Bergstrom M, Litton J, Eriksson L, Bohm C, Blomqvist G. Determination of object contour from projections for attenuation correction in cranial positron emission tomography. J Comput Assist Tomogr. 1982;6:365-372.

23. Weinzapfel BT, Hutchins GD. Automated PET attenuation correction model for functional brain imaging. J Nucl Med. 2001;42:483-491.

24. Montandon ML, Zaidi H. Atlas-guided non-uniform attenuation correction in cerebral 3D PET imaging. Neuroimage. 2005;25:278-286.

25. Rota Kops E, Herzog H. Template-based attenuation correction of PET in hybrid MR-PET scanners [abstract]. J Nucl Med. 2008;49(suppl 1):162P.

26. Catana C, van der Kouwe AJW, Benner T, et al. Rigid-body MR-assisted PET motion correction [abstract]. J Nucl Med. 2009;50(suppl 2):155P. 